\title{
COPE-28: un análisis psicométrico de la versión en español del Brief COPE*
}

COPE-28: A Psychometric Analysis of the Spanish Version of the Brief COPE

Recibido: julio 19 de 2009 ｜ Revisado: noviembre 28 de 2009 | Aceptado: enero 15 de 2010

\author{
CONSUElO MORÁN ** \\ Universidad de León, España \\ RENÉ LANDERO *** \\ Mónica Teresa GONZÁlez \\ Universidad Autónoma de Nuevo León, México
}

Para citar este artículo. Morán, C., Landero, R. \& González, M.T. (2010). COPE-28: un análisis psicométrico de la versión en español del Brief COPE. Universitas Psychologica, 9 (2), 543-552.

* Artículo de investigación psicométrica.

** Dirección a la que se dirigirán las peticiones de separatas: Universidad de León. Departamento de Psicología, Sociología y Filosofía. Facultad de Ciencias del Trabajo. Campus de Vegazana. 24071 León, España. Correo electrónico:mcmora@unileon.es

*** Cuerpo académico en Psicología Social de la Facultad de Psicología, Universidad Autónoma de Nuevo León, Mutualismo 110, Col. Mitras Centro, 64460 Monterrey, N.L., México. Tel. (81)8333-7859, fax: (81)8348-3781. Correos electrónicos: rlandero1_ mx@yahoo.com.mx; monygzz77@yahoo.com

\section{RES U MEN}

El propósito del presente estudio es analizar las propiedades psicométricas del cuestionario Brief COPE en población española, considerando la estructura y dimensiones básicas del cuestionario, llamado COPE-28 con la versión española del mismo. La muestra fue de 260 adultos. Para analizar la estructura factorial, se realizó un análisis factorial exploratorio con el método de componentes principales y rotación varimax. Posteriormente, se realizó un análisis factorial de segundo orden con las subescales que forman el COPE-28. El resultado de la prueba KMO pone en duda la factibilidad de realizar un análisis factorial; el análisis no confirma la estructura factorial original del instrumento. Con base en los resultados obtenidos se afirma que la validez del COPE-28 no es concluyente.

Palabras clave autores

Afrontamiento, brief COPE, estrés.

Palabras clave descriptores

Psicometría, estrés psicológico, conducta y mecanismos de conducta.

\section{A B S T R A C T}

The aim of this paper was to analyze psychometric properties of Spanish version of the Brief COPE questionnaire, analyzing its factor structure. The Spanish version of the Brief COPE was named COPE-28. The sample was 260 adult people. Exploratory factor analysis with principal components method and varimax rotation was used to extract the factors; as well as a second order analysis. The KMO result showed a non adequate value; nevertheless the analysis was performed and factor analysis did not confirm the factor structure of the Brief COPE. Due to the obtained results, the validity of the COPE-28 is not conclusive.

Key words authors Coping, Brief COPE, Stress.

Key words plus

Behavior and Behavior Mechanisms, Psychometrics, Stress (Psychology). 


\section{Introducción}

Las diferencias individuales bajo estrés son el resultado de los esfuerzos del afrontamiento de las personas para cambiar las situaciones estresantes, $\mathrm{O}$ para regular sus reacciones emocionales (Lazarus \& Folkman, 1984, 1986). Desde esta visión, el afrontamiento modula las diferencias que existen entre los individuos ante situaciones de estrés. Lazarus y Folkman (1986) definieron el afrontamiento como los esfuerzos cognitivos y conductuales constantemente cambiantes, que se desarrollan para manejar las demandas específicas que son valoradas como situaciones que exceden o desbordan los recursos de una persona. Un afrontamiento adecuado a una situación determinada conduce a un ajuste adecuado, y como evidencia de la adaptación encontramos el "bienestar, el funcionamiento social y la salud somática” (Lazarus, DeLongis, Folkman \& Gruen, 1985).

El afrontamiento es considerado como un factor estabilizador, de manera que facilita el ajuste individual y la adaptación cuando se está ante situaciones estresantes. El afrontamiento es cualquier esfuerzo para manejar el estrés, son las cosas que las personas hacen para evitar ser dañadas por las adversidades de la vida. Zeidner y Hammer (1990) definen el afrontamiento "como características o conductas en curso que capacitan a los individuos para manejar los estresores de forma más efectiva, experimentar pocos síntomas o que éstos sean menos severos una vez expuestos al estresor, o recuperarse más rápidamente de la exposición" (p. 694). Se denominan estrategias de afrontamiento a las acciones de los individuos tendientes a frenar, amortiguar, y si es posible anular, los efectos de las situaciones amenazantes. Sin embargo, algunos autores hablan de estilos, o recursos, además de estrategias de afrontamiento. Sandín (2003) indica que las formas de afrontamiento proporcionan pautas para explicar las diferencias en la adaptación al estrés; también tienen que ver con las diferencias que se dan entre los individuos en la mayor o menor exposición a los estresores sociales; además, hace la diferencia entre estrategias, estilos y recursos, explicando que las estrategias de afrontamiento son más específicas, se llevan a cabo acciones concretas para modificar las condiciones del estresor, por ejemplo: relajarse, ver películas, buscar apoyo social. Los estilos de afrontamiento constituyen acciones más generales, son tendencias personales para llevar a cabo una u otra estrategia de afrontamiento y los recursos de afrontamiento son características personales y sociales en las que se basa el individuo para hacer frente a los estresores, por ejemplo, "locus" de control, percepción de control, auto eficacia, autoestima, sentido del optimismo (Sandín, 2003).

Cuando Folkman y Moskowitz (2004) repasan los avances realizados en la investigación sobre afrontamiento en adultos, señalan los desafíos más importantes a los que los investigadores se tienen que enfrentar. Se trata de la clasificación de las estrategias o funciones del afrontamiento, así como también de problemas relacionados como son la medición y efectividad del afrontamiento.

Son varias las medidas de afrontamiento que más ampliamente se han utilizado, y entre éstas se incluyen: el Ways of Coping (Lazarus \& Folkman, 1984); el Multidimensional Coping Inventory (Endler \& Parker, 1990); y, el Inventario COPE (Carver, Scheier \& Weintraub, 1989), entre otras.

La versión original del Ways of Coping (WOC) se desarrolló como medición general del afrontamiento, era el Ways of Coping Checklist (Folkman \& Lazarus, 1980) que evaluaba con 68 ítems una variedad de estrategias de afrontamiento cognitivas y conductuales, con dos respuestas alternativas (sí y no). Estos 68 ítems los generaron los autores a partir de la literatura sobre el tema y por deducción de su propia teoría. Los ítems así elaborados fueron clasificados en dos escalas: en la primera estaban las estrategias centradas en la resolución del problema y, en la segunda, las centradas en regular el malestar emocional ante la situación problemática. El instrumento fue revisado sucesivamente. Los estudios de validez y fiabilidad fueron realizados en la mitad de la década de 1980. Las propiedades psicométricas del cuestionario eran muy limitadas, ya que se basaban en varios estudios realizados con la primera versión del instrumento, que ya no está en uso. A esta primera versión del WOC se le hi- 
cieron sucesivas revisiones (Folkman \& Lazarus, 1984) el cuestionario quedó con 66 ítems. El análisis de la estructura factorial de esta nueva versión realizada por Folkman, Lazarus, Dunkel-Scheter, DeLongis y Gruen (1986) reveló la existencia de ocho dimensiones, denominadas: confrontación, distanciamiento, autocontrol, búsqueda de apoyo social, aceptación de la responsabilidad, escapeevitación, planificación y reevaluación positiva. Esta versión ha sido la más utilizada en español y su estructura factorial ha sido obtenida en otros estudios, en España (Aliaga \& Capafóns, 1996; Sánchez-Cánovas, 1991). Posteriormente, se volvió a revisar en 1985, 1986 y finalmente en 1988. La última versión tiene 50 ítems (Folkman \& Lazarus, 1988) y se le realizaron algunos cambios de estilo, así como la eliminación de ítems con menor peso en cada factor. Existen estudios sobre esta versión que ponen en duda la estabilidad de su estructura factorial (Olmedo, Ibáñez \& Hernández, 2001).

Siguiendo el modelo teórico del estrés de Lazarus, otros investigadores (Carver, Scheier \& Weintraub, 1989) propusieron el COPE como alternativa al Ways of Coping. El COPE pretende ser un instrumento más preciso en la formulación de los ítems, así como ampliar el número de áreas a evaluar, incluyendo algunas que no estaban en el anterior.

El COPE es un inventario multidimensional, desarrollado para evaluar las diferentes formas de respuesta ante el estrés. Cinco de sus escalas (de cuatro ítems cada una) miden conceptualmente distintos aspectos del afrontamiento centrado en el problema (afrontamiento activo, planificación, supresión de actividades distractoras, refrenar el afrontamiento, búsqueda de apoyo social instrumental). Las cinco escalas miden aspectos que pueden ser considerados como afrontamiento centrado en la emoción (búsqueda de apoyo social emocional, reinterpretación positiva, aceptación, negación, volver a la religión); y tres escalas miden respuestas de afrontamiento que posiblemente son menos utilizadas (deshago emocional, desconexión emocional, desconexión mental) (posteriormente se añadieron uso de drogas y alcohol y humor) (Carver, 1991, documento no publicado). De esta manera, en su versión final queda un instrumento de 60 ítems y 15 escalas. Varias de ellas están basadas en discusiones teóricas específicas acerca del carácter funcional, o potencialmente menos funcional, de las propiedades de las estrategias de afrontamiento. Otras escalas fueron incluidas porque investigaciones previas indicaron que las tendencias del afrontamiento pueden reflejar cualquiera de las dos formas, puede ser que aumente o puede impedir el afrontamiento adaptativo. El inventario COPE ha sido adaptado en España por Crespo y Cruzado (1997).

Más adelante Carver (1997) presenta una versión abreviada del anterior COPE, que es utilizada en investigaciones relacionadas con la salud. Es el Brief COPE, que consta de 14 subescalas, de dos ítems cada una. Esta versión abreviada omite dos subescalas del COPE completo (supresión de actividades distractoras y refrenar el afrontamiento) ya que no fue confirmada su validez en los trabajos previos. Otras tres subescalas fueron ligeramente reenfocadas debido a que habían demostrado ser problemáticas en los estudios precedentes. A la subescala originalmente denominada reinterpretación positiva y crecimiento, se le denomina solamente reinterpretación positiva. Finalmente, la subescala llamada humor, no forma parte del inventario original COPE y fue añadida por Carver, porque se consideró que era evidente la importancia de esta respuesta.

Los ítems son planteados en términos de la acción y la respuesta que las personas realizan en una escala ordinal con cuatro alternativas (de $\mathrm{O}$ a 3), entre "nunca hago esto" hasta "siempre hago esto". Esta respuesta puede ser cambiada según que el investigador esté interesado en el afrontamiento estado o en el afrontamiento rasgo. En el primer caso deberá responder "hice esto" (en relación a una situación dada de antemano), para la opción rasgo se deberá responder "acostumbro a hacer esto".

Su creador, Carver (1997), considera que es idóneo en investigación, ya que proporciona una medida breve de afrontamiento que evalúa las variadas respuestas conocidas relevantes para un afrontamiento efectivo o ineficaz. Lo desarrolló con la intención de reducir la sobrecarga y el 
esfuerzo que tenían que realizar al responder al inventario completo. Escogió 2 ítems por escala, aquellos que habían tenido un peso mayor en análisis factorial previo, por la claridad, y por el sentido del ítem para los respondientes.

El Brief COPE consta de las siguientes subescalas: (se añade una breve descripción).

1. Afrontamiento activo: Iniciar acciones directas, incrementar los propios esfuerzos eliminar o reducir al estresor.

2. Planificación: Pensar acerca de cómo afrontar al estresor. Planificar estrategias de acción, los pasos a dar y la dirección de los esfuerzos a realizar.

3. Apoyo instrumental: Procurar ayuda, consejo, información a personas que son competentes acerca de lo que debe hacer.

4. Uso de apoyo emocional: Conseguir apoyo emocional de simpatía y de comprensión.

5. Auto-distracción: Concentrarse en otros proyectos, intentando distraerse con otras actividades, para tratar de no concentrarse en el estresor.

6. Desahogo: Aumento de la conciencia del propio malestar emocional, acompañado de una tendencia a expresar o descargar esos sentimientos.

7. Desconexión conductual: Reducir los esfuerzos para tratar con el estresor, incluso renunciando al esfuerzo para lograr las metas con las cuales se interfiere al estresor.

8. Reinterpretación positiva: Buscar el lado positivo y favorable del problema e intentar mejorar o crecer a partir de la situación.

9. Negación: Negar la realidad del suceso estresante.

10. Aceptación: Aceptar el hecho de lo que está ocurriendo, de que es real.

11. Religión: La tendencia a volver hacia la religión en momentos de estrés, aumentar la participación en actividades religiosas.

12.Uso de sustancias (alcohol, medicamentos): Significa tomar alcohol u otras sustancias con el fin sentirse bien o para ayudarse a soportar al estresor.
13. Humor: Hacer bromas sobre el estresor o reírse de las situaciones estresantes, haciendo burlas de la misma.

14. Auto-inculpación: Criticarse y culpabilizarse por lo sucedido.

15.El objetivo de este trabajo es analizar las propiedades psicométricas de la versión en español del inventario Brief COPE de Carver (1997), en población española. De forma más específica, el estudio se plantea la determinación de la estructura y dimensiones básicas del inventario en la muestra española de este estudio.

\section{Método}

\section{Participantes}

Participaron en el estudio 260 personas con edad promedio de 37.4 años $(D T=9.8)$, el $50.8 \%$ se encuentran en el rango de 21 a 36 años, $40.1 \%$ de 37 a 50 años, y de 51 a 61 el 9.1\%. La distribución por sexo es $66.8 \%$ mujeres y $33.2 \%$ hombres. De ellos el 8\% tenía formación básica; el 19\%, bachillerato; el 55\%, estudios universitarios (un 18\% no informaron de su nivel académico). El 37\% eran estudiantes universitarios de la Facultad de Ciencias del Trabajo y de Doctorado de la Universidad de León y el 63\% trabajadores del sector de la enseñanza (profesores, administrativos, monitores, etc.).

\section{Instrumentos}

COPE-28 es la versión española del Brief COPE de Carver (1997) realizada por Morán y Manga (documento sin publicar). Es un inventario de 28 ítems y 14 subescalas que se responde en una escala ordinal tipo Likert de 4 alternativas de respuesta (de 0 a 3 ), entre "nunca hago esto" a "hago siempre esto" con puntuaciones intermedias.

\section{Procedimiento}

El Brief COPE fue traducido al español teniendo en cuenta la equivalencia léxica y gramatical, así como su equivalencia conceptual y cultural, ade- 
más se consideró el emparejamiento del contenido del ítem con la dimensión que se quiere evaluar. La traducción se realizó por dos investigadores, llegando a una versión de consenso. Después se aplicó la versión final a una muestra de sujetos para que realizaran sugerencias sobre cómo percibían las preguntas y el formato de respuesta.

Los estudiantes universitarios, respondieron al cuestionario en el contexto del aula, donde los grupos tenían menos de 50 alumnos. Asimismo, se pidió la colaboración voluntaria de profesores no universitarios y de otros trabajadores en varios centros de enseñanza de la ciudad de León (España), quienes rellenaron el cuestionario en su tiempo libre, en el lugar de trabajo. En todos los casos la aplicación fue realizada por psicólogos y psicopedagogos.

\section{Procedimiento de análisis estadístico}

Para analizar la estructura factorial del COPE-28, se realizó en primer lugar un análisis factorial exploratorio (AFE) con el método de componentes principales (PCA) y rotación varimax. Posteriormente, se realizó un análisis factorial de segundo orden con las subescales que forman el COPE-28 y se estimaron las correlaciones entre las formas de afrontamiento.

A pesar de que el análisis de consistencia interna no es recomendable para subescalas formadas por dos ítems, se incluye el valor del alfa de Cronbach como referencia para el lector. Asimismo, se estimó el alfa de Cronbach para los ítems que formaron cada factor en el análisis factorial de segundo orden, donde fue necesario invertir las puntuaciones en el caso de los ítems con carga factorial negativa en el factor 1 .

\section{Resultados}

\section{Correlación entre ítems}

Como se mencionó, el análisis de fiabilidad de cada subescala no es el más adecuado, ya que las subescalas están formadas solamente por 2 ítems; sin embargo, a manera de referencia, se presenta en la Tabla 1, donde puede observarse que los valores del alfa de Cronbach son bajos en la mayoría de las subescalas. Asimismo, se presentan los resultados de la correlación entre los 2 ítems que forman cada subescala, todas las correlaciones son positivas y significativas; sin embargo, la correlación entre los ítems que forman aceptación aunque es significativa, es débil comparada con los otros ítems, además, su consistencia interna no es aceptable (Hair, Anderson, Tatham \& Black, 1999).

\section{TABLA 1}

Correlación entre los ítems de cada subescala del CO. PE-28 y valores de consistencia interna

\begin{tabular}{|c|c|c|c|}
\hline & $\begin{array}{l}\text { Correla- } \\
\text { ción }\end{array}$ & $\begin{array}{l}\text { Alfa de } \\
\text { Cronbach }\end{array}$ & $\begin{array}{c}\text { Alfa de } \\
\text { Cronbach } \\
\text { (Carver, } \\
\text { 1997) }\end{array}$ \\
\hline $\begin{array}{l}\text { Apoyo } \\
\text { Emocional }\end{array}$ & $.642 * *$ & .78 & .71 \\
\hline Apoyo Social & $.472 * *$ & .64 & .64 \\
\hline $\begin{array}{l}\text { Afrontamiento } \\
\text { Activo }\end{array}$ & $.403 * *$ & .58 & .68 \\
\hline Planificación & $.433 * *$ & .60 & .63 \\
\hline $\begin{array}{l}\text { Uso de } \\
\text { Sustancias }\end{array}$ & $.868 * *$ & .93 & .90 \\
\hline Humor & $.655 * *$ & .79 & .73 \\
\hline Religión & $.662 * *$ & .80 & .82 \\
\hline $\begin{array}{l}\text { Autodistrac- } \\
\text { ción }\end{array}$ & $.420 * *$ & .59 & .71 \\
\hline Negación & $.469 * *$ & .64 & .54 \\
\hline Desahogo & $.409 * *$ & .58 & .50 \\
\hline $\begin{array}{l}\text { Autoinculpa- } \\
\text { ción }\end{array}$ & $.403 * *$ & .58 & .69 \\
\hline Desconexión & $.459 * *$ & .63 & .65 \\
\hline $\begin{array}{l}\text { Reinterpreta- } \\
\text { ción Positiva }\end{array}$ & $.423 * *$ & .59 & .64 \\
\hline Aceptación & $.176^{* * *}$ & .30 & .57 \\
\hline
\end{tabular}

$* p<.05, * * p<.01$.

Fuente: elaboración propia. 


\section{Análisis factorial exploratorio}

Para la primera etapa del AFE, el valor correspondiente a la prueba de Kaiser-Meyer-Olkin (KMO) fue de .663 y la prueba de esfericidad de Bartlett fue significativa $\left(\chi^{2}=2290.052 ; g l=378 ; p=.001\right)$.

Como se pretendía confirmar la estructura factorial del COPE-28, se realizó el AFE restringiendo a 14 factores, confirmando la distribución de ítems en 11 subescalas, al tomar como criterio de inclusión un peso factorial mayor o igual a .40 (Hair, Anderson, Tatham \& Black, 1999). Los ítems que teóricamente forman las subescalas de apoyo emocional y apoyo social se agruparon en un solo factor; además el ítem 1 que forma parte de la subescala de apoyo social, carga en el factor 13, aparentemente residual. En el factor 14, solo carga un ítem.

Al intentar una solución de 13 factores, no mejora la interpretación, por lo que se probó una solución de 12 factores encontrando que apoyo social y apoyo emocional forman un solo factor, lo mismo que afrontamiento activo y planificación. El ítem 3 que forma parte de la subescala de aceptación carga en tres factores con valores entre 31 y.34. Al eliminar el ítem 3, el ítem 21 (el otro que forma la subescala de aceptación) carga en el último factor, aparentemente residual. Al eliminarlo (lo que da como resultado eliminar del análisis la subescala de aceptación) y restringir el AFE a 11 factores, se confirma la distribución de ítems en 9 de las subescalas del COPE-28; los ítems que componen apoyo social y apoyo emocional forman un solo factor, lo mismo que los ítems que forman planificación y afrontamiento activo (Tabla 2). La varianza explicada por estos 11 factores es de $74.29 \%$.

TABLA 2

Cargas factoriales de los ítems del COPE-28*

Carga factorial

\begin{tabular}{rr}
\hline Factor 1 & \\
9 apoyo emocional & .756 \\
17 apoyo emocional & .801
\end{tabular}

\begin{tabular}{lc}
\hline & Carga factorial \\
\hline 1 apoyo social & .686 \\
28 apoyo social & .853
\end{tabular}

Factor 2

2 Afrontamiento activo $\quad .726$

10 Afrontamiento activo $\quad .644$

6 Planificación $\quad .678$

26 planificación $\quad .718$

Factor 3

15 uso de sustancias $\quad 930$

24 uso de sustancias $\quad .929$

\section{Factor 4}

7 humor $\quad .885$

19 humor $\quad .836$

Factor 5

16 religión $\quad .876$

20 religión $\quad .905$

Factor 6

4 autodistracción $\quad .831$

22 autodistraccion $\quad .789$

Factor 7

5 negación $\quad .745$

13 negación $\quad .847$

Factor 8

12 desahogo $\quad .804$

23 desahogo $\quad .783$

Factor 9

8 autoinculpación $\quad .809$

27 autoinculpación $\quad .773$

Factor 10

11 desconexión $\quad .870$

25 desconexión $\quad .724$

Factor 11

14 reinterpretación positive $\quad .873$

18 reinterpretación positive $\quad .595$

*Se incluye el número de ítem y la subescala a la que pertenece teóricamente.

Fuente: elaboración propia. 
La eliminación de la subescala aceptación en el AFE, se respalda en la débil correlación entre los dos ítems que la forman $(r=.176)$ y por lo señalado anteriormente. Es decir, no hay suficiente evidencia que justifique conservarlos para el análisis realizado.

\section{Análisis factorial de segundo orden}

Se realizó un análisis factorial con las puntuaciones de cada subescala. El valor correspondiente a la prueba de Kaiser-Meyer-Olkin (KMO) fue de .654 y la prueba de esfericidad fue significativa $\left(\chi^{2}=\right.$ 482.986; $g l=78 ; p=.001)$. El número de factores sugerido por el criterio de autovalor mayor a 1 (60.87\% de varianza explicada) fue de 5 , mientras que el gráfico de sedimentación sugiere 4 factores.

Se probaron las soluciones con 4 y 5 factores, siendo más interpretable la solución de 4 factores, donde las subescalas se agrupan en afrontamiento cognitivo, bloque del afrontamiento (evitación), afrontamiento mediante apoyo social y afrontamiento espiritual (Tabla 3). Para este análisis, se omitió la subescala de aceptación, el porcentaje de varianza explicada por 4 factores fue de $52.99 \%$. Se estimó el alfa de Cronbach para cada factor, para afrontamiento cognitivo y afrontamiento de apoyo social el alfa fue de .74; para bloqueo del afrontamiento fue .71; mientras que para afrontamiento espiritual, formado únicamente por los dos ítems de religión, el alfa fue de .80 .

\section{Discusión}

Para el presente estudio, se planteó el objetivo de analizar las propiedades psicométricas del inventario Brief COPE de Carver (1997); en el proceso se presentaron algunas dificultades, por lo que la validez del instrumento no es concluyente, no se confirma la estructura factorial original; además,

TABLA 3

Análisis factorial de orden superior para el COPE-28

\begin{tabular}{|c|c|c|c|c|}
\hline & $\begin{array}{l}\text { Afrontamiento } \\
\text { cognitivo } \\
\alpha=.74\end{array}$ & $\begin{array}{l}\text { Afrontamiento de } \\
\text { apoyo social } \\
\alpha=.74\end{array}$ & $\begin{array}{c}\text { Bloqueo del } \\
\text { afrontamiento } \\
\alpha=.71\end{array}$ & $\begin{array}{c}\text { Afrontamiento } \\
\text { espiritual } \\
\alpha=.80\end{array}$ \\
\hline Afrontamiento activo & .759 & & & \\
\hline Planificación & .676 & & & \\
\hline Apoyo emocional & & .782 & & \\
\hline Apoyo social & & .793 & & \\
\hline Religión & & & & .868 \\
\hline Reinterpretación positiva & .410 & & & \\
\hline Negación & & & .592 & \\
\hline Humor & .420 & & .550 & \\
\hline Autodistracción & & & .522 & \\
\hline Autoinculpación & & & .475 & \\
\hline Desconexión & -.645 & & & \\
\hline Desahogo & & .584 & & \\
\hline Uso de sustancias & & & .692 & \\
\hline
\end{tabular}

Fuente: elaboración propia. 
la consistencia interna de las subescalas no es adecuada en todos los casos.

El valor de la prueba de KMO no es el más adecuado, lo que pone en duda la factibilidad de realizar un análisis factorial para los ítems que forman el COPE-28. En cuanto a la estructura factorial, al igual que en el trabajo de Carver (1997), los ítems que forman las subescalas de apoyo social y apoyo emocional forman un solo factor; asimismo, los ítems que se incluyen en afrontamiento activo y planificación cargan en el mismo factor. Mientras que los ítems que forman la subescala de aceptación no presentan un comportamiento adecuado en el análisis factorial.

Estadísticamente ésta sería la mejor estructura del instrumento, se recomienda que se confirme en otras muestras y que para fines de investigación se contemple la posibilidad de utilizar 11 subescalas y no 14 , como se plantea originalmente. Sin embargo, en casos donde sea necesario contar con medidas de cada una de las 14 formas de afrontamiento propuestas teóricamente para la escala, convendría utilizar la puntuación de cada subescala respetando los planteamientos teóricos, ya que se trabaja con indicadores de cada forma de afrontamiento que pudieran ser relativamente independientes entre sí.

En cuanto al análisis factorial de segundo orden, las subescalas se agrupan lógicamente en tipos de afrontamiento más generales. Cabe mencionar que los resultados de este estudio tienen limitaciones, debido en parte al tipo de muestra, y a no contar con otras medidas para estimar validez de criterio. Por lo que se recomienda continuar con otros estudios donde se analice este instrumento.

Consideramos de interés mencionar que se han dado diferentes nombres a las formas de afrontamiento dependiendo del instrumento utilizado para su medición, lo que dificulta la comparación entre las diferentes escalas diseñadas. Tomando en cuenta los resultados de este estudio y de estudios como el de Olmedo et al. (2001), donde se ponen de manifiesto las diferencias en la estructura factorial de escalas de afrontamiento al pasar de una muestra a otra. Es por esto que recomendamos que para poblaciones específicas (estudiantes, personas con alguna enfermedad crónica, etc.), se diseñen instrumentos que reflejen las formas comunes de afrontamiento utilizadas por esa población, es decir, por personas que comparten ciertas características.

\section{Referencias}

Aliaga F. \& Capafóns, A. (1996). La medida del afrontamiento: revisión crítica de la "Escala de Modos de Coping" (Ways of Coping). Ansiedad y Estrés, 2(1), 17-26.

Bermúdez, J. (1996). Afrontamiento: aspectos generales. En A. Fierro (Comp.), Manual de psicología de la personalidad (pp. 289-209). Barcelona: Paidós.

Blanch, A., Aluja, A. \& Biscarri, J. (2000). Burnout syndrome and coping strategies: A structural relations model. Revista de Psicología del Trabajo y de las Organizaciones, 18(1), 57-74.

Carver, C. S. (1997). You want to measure coping but your protocol's too long: Consider the Brief COPE. International Journal of Behavioral Medicine, 4(1), 92-100.

Carver, C. S., Scheier, M. F. \& Weintraub, J. K. (1989). Assessing coping strategies: A theoretically based approach. Journal of Personality and Social Psychology, 56, 267-283.

Crespo, M. \& Cruzado, J. A. (1997). La evaluación del afrontamiento: adaptación española del cuestionario COPE con una muestra de estudiantes universitarios. Análisis y Modificación de Conducta, 23(92), 797-830.

Endler, N. S. \& Parker, J. D. A. (1990). Multidimensional assessment of coping: A critical evaluation. Journal of Personality and Social Psychology, 58, 844-854.

Folkman, S. \& Lazarus, R. S. (1988). Ways of Coping Questionnaire: Research edition manual. Palo Alto, CA: Consulting Psychologists Press.

Folkman, S. \& Moskowitz, J. T. (2000). Positive affect and the other side of coping. American Psychologist, 55, 647-654.

Folkman, S. \& Moskowitz, J. T. (2004). Coping: Pitfalls and promise. Annual Review of Psychology, 55, 745-774. 
Folkman, S., Lazarus, R. S., Dunkel-Scheter, C., DeLongis, A. \& Gruen, R. J. (1986). Dynamics of stressful encounter: Cognitive appraisal, coping, and encounter outcomes. Journal of Personality and Social Psychology, 50, 992-1003.

Hair, J., Anderson, R., Tatham, R. \& Black, W. (1999). Análisis multivariante. España: Prentice Hall.

Lazarus, R. S. (2000). Toward better research on stress and coping. American Psychologist, 55, 665-673.

Lazarus, R. S. (2006). Emotions and interpersonal relationships: Toward a person-centered conceptualisation of emotions and coping. Journal of Personality, 74(1), 9-46.

Lazarus, R. S., DeLongis, A., Folkman, S. \& Gruen, R. (1985). Stress and adaptational outcomes: The problem of confounded measures. American Psychologist, 40, 770-779.

Lazarus, R. S. \& Folkman, S. (1984). Stress, appraisal and coping. Nueva York: Springer Publishing Company.

Lazarus, R. S. \& Folkman, S. (1986). Estrés y procesos cognitivos. Barcelona: Martínez Roca.

McElfatrick, S., Carson, J., Annett, J., Cooper, C. L., Holloway, F. \& Kuipers, E. (2000). Assessing coping skills in mental health nurses: Is an occupational specific measure better than a generic coping skills scale? Personality and Individual Differences, 28, 965-976.

Olmedo, E., Ibáñez, I. \& Hernández, L. (2001). Validación estructural del Ways of Coping Questionnaire de Lazarus y Folkman: un análisis factorial restrictivo. Ansiedad y Estrés, 7(1), 15-28.

Sánchez-Cánovas, J. (1991). Evaluación de las estrategias de afrontamiento. En G. Buela-Casals \& V. E. Caballo (Eds.), Manual de Psicología Clínica Aplicada (pp. 247-270). Madrid: Siglo XXI.

Sánchez-Cánovas, J. (1993). Estrategias de afrontamiento. En J. Sánchez-Cánovas \& M. P. SánchezLópez (Eds.), Psicología diferencial: diversidad e individualidad humanas Prácticas (pp. 483-525). Madrid: Centro de Estudios Ramón Areces.

Sandín, B. (2003). El estrés: un análisis basado en el papel de los factores sociales. Revista Internacional de Psicología Clínica y de la Salud, 3(1), 141-157.

Zeidner, M., \& Hammer, A. (1990). Life events and coping resources as predictors of stress symptoms in adolescents. Personality and Individual Differences, 11, 693-703.

\section{Anexo}

\section{COPE-28 (Según el original de Carver, 1997)}

Traducido por Consuelo Morán y Dionisio Manga

INSTRUCCIONES. Las frases que aparecen a continuación describen formas de pensar, sentir o comportarse, que la gente suele utilizar para enfrentarse a los problemas personales o situaciones difíciles que en la vida causan tensión o estrés. Las formas de enfrentarse a los problemas, como las que aquí se describen, no son ni buenas ni malas, ni tampoco unas son mejores o peores que otras. Simplemente, ciertas personas utilizan más unas formas que otras. Ponga 0, 1, 2 ó 3 en el espacio dejado al principio, es decir, el número que mejor refleje su propia forma de enfrentarse a ello, al problema. Gracias.

1. Intento conseguir que alguien me ayude o aconseje sobre qué hacer.

2. Concentro mis esfuerzos en hacer algo sobre la situación en la que estoy.

3. Acepto la realidad de lo que ha sucedido.

4. ___ Recurro al trabajo o a otras actividades para apartar las cosas de mi mente.

5. ___ Me digo a mí mismo "esto no es real".

6. Intento proponer una estrategia sobre qué hacer.

7. Hago bromas sobre ello.

8. _ Me critico a mí mismo.

9. Consigo apoyo emocional de otros.

10.___ Tomo medidas para intentar que la situación mejore.

11.__ Renuncio a intentar ocuparme de ello.

12. __ Digo cosas para dar rienda suelta a mis sentimientos desagradables.

13. Me niego a creer que haya sucedido.

14. Intento verlo con otros ojos, para hacer que parezca más positivo.

15._Utilizo alcohol u otras drogas para hacerme sentir mejor. 
16. Intento hallar consuelo en mi religión o creencias espirituales.

17.___ Consigo el consuelo y la comprensión de alguien.

18. ___ Busco algo bueno en lo que está sucediendo.

19. Me río de la situación.

20. Rezo o medito.

21.___ Aprendo a vivir con ello.

22. ___ Hago algo para pensar menos en ello, tal como ir al cine o ver la televisión.

23. Expreso mis sentimientos negativos.

24. Utilizo alcohol u otras drogas para ayudarme a superarlo.

25.___ Renuncio al intento de hacer frente al problema.

26.___ Pienso detenidamente sobre los pasos a seguir.

27. Me echo la culpa de lo que ha sucedido.

28. Consigo que otras personas me ayuden o aconsejen.

\begin{tabular}{|c|c|c|c|c|c|}
\hline Estrategia & ítem & Punt. & ítem & Punt. & Total \\
\hline $\begin{array}{l}\text { Afrontamiento } \\
\text { activo }\end{array}$ & 2 & & 10 & & \\
\hline Planificación & 6 & & 26 & & \\
\hline Apoyo emocional & 9 & & 17 & & \\
\hline Apoyo social & 1 & & 28 & & \\
\hline Religión & 16 & & 20 & & \\
\hline $\begin{array}{l}\text { Reevaluación } \\
\text { positiva }\end{array}$ & 14 & & 18 & & \\
\hline Aceptación & 3 & & 21 & & \\
\hline Negación & 5 & & 13 & & \\
\hline Humor & 7 & & 19 & & \\
\hline Autodistracción & 4 & & 22 & & \\
\hline Autoinculpación & 8 & & 27 & & \\
\hline Desconexión & 11 & & 25 & & \\
\hline Desahogo & 12 & & 23 & & \\
\hline Uso de sustancias & 15 & & 24 & & \\
\hline
\end{tabular}

